\title{
¿Es importante perder peso en tiempos de covid-19?*
}

\author{
[Artículos]
}

\author{
Andrés Mármol Pérez ${ }^{* *}$ \\ Salvador Baena Morales ${ }^{* * *}$ \\ Moisés Falces Prieto**** \\ Francisco Tomás González-Fernández ${ }^{* * * * *}$
}

Recibido: 17 de noviembre de 2020

Aceptado: o7 de diciembre de 2020

Citar como:

Mármol-Pérez, A., Baena-Morales, S., Falces-Prieto, M. y González-Fernández, F.T.

(2020). ¿Es importante perder peso en tiempos de covid-19? Revista de

Investigación Cuerpo, Cultura y Movimiento, 11(1).

https://doi.org/10.15332/2422474X.6465

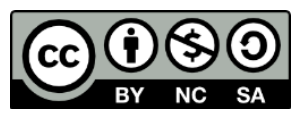

\footnotetext{
* Artículo de investigación. El presente estudio no recibió financiación para su realización. El estudio se inició de forma transversal en varias universidades españolas y se ha gestionado mediante el grupo de investigación SER: Salud, Educación y Rendimiento, Palma de Mallorca, España.

** Graduado en Ciencias de la Actividad Física y el Deporte. Huelva, España. Correo electrónico: andres_mp_97@hotmail.com; ORCID: https://orcid.org/0000-0002-64381573

*** Departamento de Didáctica General y específica. Facultad de Ciencias de la Educación. Universidad de Alicante, Alicante, España. Correo electrónico: salvador.baena@ua.es; ORCID: https://orcid.org/0000-0002-6722-371 ${ }_{* * * *}$ Departamento de Optimización del Rendimiento Físico e Investigación. Academia Marcet. Barcelona, España. Correo electrónico: mfalpri@gmail.com; ORCID: https://orcid.org/0000-0003-1828-4614 ${ }_{* * * * *}$ Doctor internacional en Biomedicina. Profesor e investigador en la Universidad Pontificia de Comillas. CESAG, Mallorca (España). Correo electrónico: ftgonzalez@comillas.edu; ORCID: https://orcid.org/0000-0002-1480-7521
} 


\section{Resumen}

La obesidad es una enfermedad que lidera el disfuncionamiento de numerosos órganos. El alto contenido adiposo conlleva a un estado inflamatorio crónico y las reservas suficientes para la hipertrofia de los adipocitos. Si se combina el efecto del covid-19 con la obesidad su severidad incrementa. Ambos resultados combinados son enormemente peligrosos y disminuyen la esperanza de vida. Incluso, la atención a las personas con obesidad en los hospitales se ve perjudicada, lo que difículta su cuidado y requiere de más personal. Las necesidades de ventilación mecánica de las personas con obesidad son previsiblemente incrementadas y esto puede ser crucial, ya que no todos los hospitales disponen de este material. Por tanto, ejercitarse mediante la combinación del entrenamiento de fuerza y de resistencia gana otra razón en estos días para combatir el virus. Añadir una restricción calórica también es otro factor clave para conseguir efectos significativos.

Palabras clave: covid-19, obesidad, ejercicio físico, tratamiento, esperanza de vida

\section{Is it important to lose weight in time of COVID-19?}

\section{Abstract}

Obesity is a disease that leads to dysfunction of many organs. High adipose content leads to a chronic inflammatory condition and sufficient reserves for adipocyte hypertrophy. If the effect of COVID-19 is combined with obesity its severity increases. Both results combined are extremely dangerous and decrease life expectancy. Even the care of people with obesity in hospitals is affected, making their care more difficult and requiring more staff. The mechanical ventilation needs of people with obesity are predictably increased and this can be crucial, as not all hospitals have this equipment. Therefore, exercising by combining strength and endurance training is another reason these days to fight the 
virus. Adding caloric restriction is also another key factor to achieve significant effects.

Keywords: COVID-19, obesity, physical exercise, treatment, life expectancy.

\section{Introducción}

Tras sufrir la etapa más difícil de nuestras vidas, la Tierra ha sido capaz de comprobar si estábamos prepararos para enfrentarnos a algo así. Con una alta prevalencia de obesidad alrededor del mundo, el covid-19 no le ha dado tiempo al sistema sanitario de reaccionar para contrarrestar los efectos de la crisis. Está claro que nadie esperaba la llegada de un virus de la magnitud del covid-19, pero podríamos haber estado más preparados si hubiéramos puesto más atención a la evidencia científica y a los virus que se presentaron previamente. Además, la industria alimenticia se ha centrado únicamente en su propio beneficio económico, lo que ha empeorado la situación de salud de la población a largo plazo (Tan et ál., 2020). Sin embargo, como punto positivo, si combinamos la unión social con las ayudas de los gobiernos, las cadenas televisivas, la industria alimenticia y la salud mundial, la situación podría mejorar.

Aunque la vacuna contra el covid-19 no se ha creado, siempre hemos tenido a nuestro alcance una píldora mágica llamada ejercicio físico, que proporciona un amplio abanico de beneficios. En términos de salud, su combinación con una restricción calórica puede ayudar a las personas con obesidad a prevenir el contagio del virus y ofrecerles un sistema inmunitario más eficiente. Si el programa de ejercicio físico está basado en la unión del entrenamiento de fuerza y el de resistencia, los pacientes tendrán la mejor combinación para luchar contra el virus. El ejercicio físico funciona como una medicina y necesita reconocerse como un 
tratamiento coadyuvante para cualquier patología durante todas sus etapas, para que eleve los efectos potenciales de los medicamentos.

\section{Las personas con obesidad se consideran de alto riesgo en tiempo de covid-19?}

En la historia de la humanidad nunca nos hemos enfrentado a un virus como el covid-19. Desde sus comienzos en China, muchas personas lo han padecido. Al principio, la tercera edad parecería ser el grupo de población más vulnerable debido al hecho de que las dificultades respiratorias son más comunes en estas edades. No obstante, semanas más tarde, adultos jóvenes parecían contraer el virus con más facilidad y enfrentarlo con menor eficacia. En busca de la respuesta a este misterio, investigadores encontraron que la obesidad era un claro marcador de riesgo independiente, al igual que la diabetes y la hipertensión. Es bien sabido que otros virus previos también se veían afectados por grandes reservas de tejido adiposo como el covid-19 mostrado en la figura 1. Tristemente si relacionamos esta pandemia y la ya existente de la obesidad, en Estados Unidos encontramos personas con alto riesgo (Ryan et ál., 2020). Es más, el sedentarismo y la inactividad están también presentes en países como el ya mencionado, situación que aumenta los efectos negativos de la cuarentena (Chulvi-Medrano et ál., 2020)

Figura 1. Entre 124 casos positivos por covid-19, $47.6 \%$ de los pacientes eran obesos.
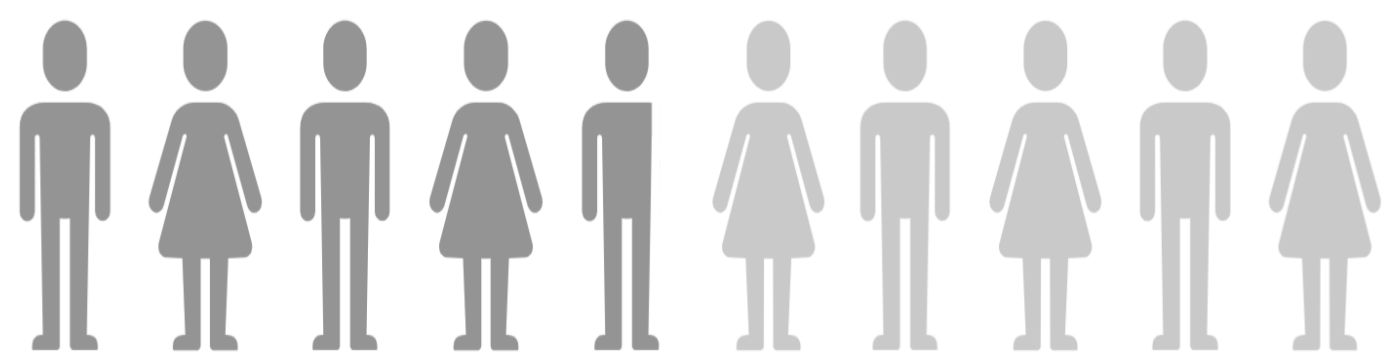

Fuente: Simonnet et ál., 2020 
Un estudio de más de 5200 pacientes hospitalizados con covid-19 señaló una fuerte asociación con la obesidad (Carter et ál., 2020). Aunque no podemos olvidar que su severidad aumenta con la edad. Sin embargo, personas menores de 60 años con sobrepeso u obesidad a menudo parecen mostrar el doble de hospitalizaciones. Esto significa que la obesidad trae consigo un riesgo mayor de contraer enfermedades infecciosas (Hudson y Sprow, 2020), y, adicionalmente, un incremento del peso puede llevar a serias consecuencias de salud como una rápida pérdida de la capacidad cardiorrespiratoria y el Enterococos resistentes a la Vancomicina. Por lo tanto, los pacientes obesos con covid-19 deben tratarse como población de alto riesgo (Petrilli et ál., 2020).

Por un lado, el covid-19 tiene una gran afinidad con la encima convertidora de angiotensina II (ACE2) y su expresión es más alta en el tejido adiposo. Incluso, como la función de ACE2 de convertir angiotensina 2 (AGT2) en angiotensina 1-7 (AGT1-7) es inhibida, la actividad de AGT2 se ve incrementada provocando una respuesta inflamatoria y un consecuente incremento de la permeabilidad vascular. Por ello, las personas con obesidad parecen tener más posibilidad de contraer el virus que las personas con normopeso (Kassir, 2020).

Por otro lado, no solo la obesidad facilita contraer el virus, sino que afecta el sistema inmunitario provocando una pobre respuesta ante el covid-19. El desequilibrio metabólico de una persona con obesidad compromete al sistema inmunitario aumentando el riesgo de contraer enfermedades respiratorias. Adicionalmente, la función de las células T y los macrófagos no es la esperada y las vacunas tienen un menor efecto (Maffetone y Laursen, 2020). En otras palabras, la probabilidad de sobrevivir siendo obeso es mucho menor que la de las personas con normopeso, puesto que su severidad y letalidad es muy alta. 
Teniendo todo en consideración, podemos empezar a preocuparnos por aquellos que siempre han estado en riesgo. Sin ninguna duda, los gobiernos y sistemas sanitarios tendrán que ejecutar estrategias para mitigar esta emergencia, sobre todo durante la fase de prevención y tratamiento.

\section{Perder peso es un factor clave para prevenir y contrarrestar los efectos del covid-19?}

En la actualidad, la obesidad se ha convertido en una pandemia muy difícil de erradicar alrededor del mundo. Muchas organizaciones se están beneficiando de ellos y algunos gobiernos no están tomando cartas en el asunto, aun sabiendo que la obesidad aumentó el riesgo de mortalidad y morbilidad tras infecciones virales como el covid-19 (Misumi et ál., 2019).

Con frecuencia, se proponen numerosos métodos para perder peso. Aun así, lo que es una evidencia es que sin un déficit calórico es muy complicado. Según el Colegio Americano de Medicina del Deporte (ACSM, por sus siglas en inglés), la restricción calórica ha de ser en torno a 300 kilocalorías diarias. Indudablemente, el apoyo de nutricionistas se requerirá para individualizar cada caso porque la restricción no puede ser más alta debido al posible efecto revote, que hace que se recupere el peso perdido o incluso se gane más tras la etapa de restricción.

Tabla 1. Beneficios de perder peso para prevenir y contrarrestar los efectos del covid-19

- Menor permeabilidad vascular para dificultar el síndrome respiratorio agudo severo.

- $\quad$ Sistema inmunológico más saludable (4)

- Estado inflamatorio no crónico

- Mayor volumen de reserve respiratoria

Fuente: elaboración propia. 
Aunque la obesidad llegó a ser una emergencia pública hace muchos años, todavía existe la necesidad apremiante de resolverla.

\section{Prescripción de ejercicio para perder peso}

En ninguna circunstancia, el ejercicio físico ha de dejarse a un lado en un programa de pérdida de peso, ya que las reducciones son mayores. Uno de los factores clave es aumentar el gasto calórico diario que se puede conseguir con la combinación de una restricción calórica y el ejercicio físico. En el caso de la obesidad mórbida es necesaria la cirugía gástrica. Incluso, el descanso y el estrés deben tenerse en cuenta para conseguir el objetivo. Es ampliamente sabido que estos dos factores, entre otros, afectan al ser humano.

Siguiendo las recomendaciones del ACSM para personas con obesidad, la siguiente tabla muestra los requerimientos básicos:

Tabla 2. Pautas generales para prescribir actividad física para perder peso

\begin{tabular}{|c|c|}
\hline \multicolumn{2}{|c|}{$\begin{array}{l}\text { Sobre el objetivo: cumplir con las pautas de actividad física del ACSM teniendo en } \\
\text { cuenta lo siguiente para perder peso }\end{array}$} \\
\hline \multirow[t]{2}{*}{ Frecuencia } & Impactante: 4 días por semana \\
\hline & Superior: 5 días por semana \\
\hline \multirow[t]{2}{*}{ Duración } & Impactante: 40 minutos por sesión \\
\hline & Superior: 60 minutos por sesión \\
\hline \multirow[t]{2}{*}{ Intensidad } & Impactante: Moderada \\
\hline & Superior: De moderada a vigorosa \\
\hline
\end{tabular}

Fuente: elaboración propia.

Algunos trabajos previos han mostrado el gran valor de adecuar los niveles de actividad física durante la pandemia del covid-19. No obstante, la dosis general de ejercicio físico para este grupo de población necesita especificar la combinación de moderada y alta intensidad. Recientemente, se ha descubierto que el propio HIIT (High Intensity Interval Training) tiene beneficios increíbles, considerando los requerimientos individuales. 
$\mathrm{Al}$ analizar todas las variables, debe hallarse un criterio para las personas con obesidad, que necesitan encontrar un tipo de ejercicio físico adecuado y con el que se sientan cómodos, ya que la adherencia al programa es imprescindible. Sin duda, no hay nada más inefectivo que abandonar el programa de entrenamiento por no haber sido individualizado. Aun así, el entrenamiento de fuerza y de resistencia han de combinarse como nos ha mostrado la ciencia.

En primer lugar, el entrenamiento de resistencia comprende cualquier ejercicio aeróbico como correr, remar o montar en bicicleta, según ACSM. No importa si al principio se opta por andar en lugar de correr, siempre y cuando la intensidad se siga aumentando. Lo que realmente importa es encontrar el tipo de ejercicio físico que les apasione y les permita llevarlo a cabo durante 3 o 4 días a la semana. Si ellos no disfrutan lo que están haciendo, no seguirán poniéndolo en práctica. A pesar del carácter novedoso del covid-19, se ha encontrado que altos niveles cardiorrespiratorios proporcionan un alto perfil inmunoprotector en pacientes que lo contraigan (Zbinden-Foncea, 2020). Esta es otra de las razones por la que vale la pena tener en cuenta el ejercicio físico. En segundo lugar, aunque el entrenamiento de resistencia es el tipo de ejercicio físico que más eleva el gasto calórico, no podemos olvidarnos del entrenamiento de fuerza. Este tipo de entrenamiento no ayuda a perder peso, sin embargo, aumenta los niveles de masa muscular teniendo un mayor gasto calórico en reposo, lo que significa que el gasto calórico total se ve altamente aumentado y la cantidad de grasa corporal reducida. Sorprendentemente, las personas obesas tienen altos niveles de masa muscular debido a que tienen que lidiar con altas cargas durante el día por lo que estas deben preservarse. Este tipo de entrenamiento debe llevarse a cabo durante 2 o 3 días a la semana trabajando todos los grupos musculares con los debidos tiempos de descanso.

Revista de Investigación Cuerpo, Cultura y Movimiento 
Hay que destacar que los estudios destinados a evaluar el efecto del covid-19 sobre las personas con obesidad están en continua actualización. Por ejemplo, las últimas evidencias al respecto muestran cómo la obesidad perjudica directamente el volumen y las funciones respiratorias, lo que genera una necesidad de asistencia respiratoria avanzada, que fue significativamente mayor en los pacientes con covid-19 que tenían obesidad (Huang et ál., 2020). Por esto, la práctica de ejercicio aeróbico va a permitir un mayor desempeño cardiovascular, además sería recomendable la realización de ejercicio aeróbico de intensidad moderadabaja al aire libre, ya que esto va a producir una mayor síntesis de la vitamina D como consecuencia de la interacción entre 7-dehidrocolesterol y la radiación ultravioleta (Carter et ál., 2020). Sin embargo, se debe considerar la realización de una actividad física progresiva que evite la alta intensidad en los ejercicios realizados. Este factor es fundamental para evitar un efecto contraproducente del sistema inmune. Esto quedaría explicado por la reducción de células inflamatorias en los pulmones, una reducción de la carga patógena, así como de las inmunoglobulinas y las citoquinas antiinflamatorias en la sangre (Laddu et ál., 2020). Además, un aumento repentino de la carga de entrenamiento de personas obesas o que no están habituadas al entrenamiento, puede asociarse con trastornos inmunológicos temporales como inflamación, estrés oxidativo e incluso lesiones musculares que impidan la realización de una rutina de actividad física (Hull et ál., 2020).

Recientemente, un estudio metanalítico ha confirmado la evidencia que sugiere que la obesidad es un factor de riesgo importante de mortalidad en pacientes con covid-19 (Hussain et ál., 2020). En esta investigación se explica cómo los pacientes con obesidad tienen un sistema inmunológico defectuoso que los hace vulnerables a un tipo de infección que requiere una respuesta de inmunidad celular rápida. Por esto, la realización de 
actividad física bien estructurada, progresiva y adecuada a cada persona debe implementarse en las personas obesas y sedentarias.

Por último, es importante mantener las medidas de seguridad necesarias, aunque creamos que la situación más grave ya pasó. El adecuado distanciamiento social, al igual que la higiene personal en el uso de gimnasios y centros deportivos, son necesarios para crear un ambiente seguro. El covid-19 ha traído consigo una emergencia sanitaria internacional en la que los gobiernos deberían adoptar programas médicos nacionales, teniendo en cuanta que el ejercicio físico es una potente herramienta para reducir la probabilidad de contraer el virus, a la vez que reduce el riesgo de mortalidad tras el contagio en personas con obesidad.

\section{Referencias}

Carter, S.J., Baranauskas, M.N. y Fly, A.D. (2020) Considerations for Obesity, Vitamin D, and Physical Activity Amid the COVID-19 Pandemic. Obesity, 28 (7):1176-1177. https://doi.org/10.1002/oby.22838

Chulvi-Medrano, I., Villa-González, E., Rial Rebullido, T. y Faigenbaum, A. (2020). The Impact of COVID-19 Quarantine on Youth: From Physical Inactivity to Pediatric Depreobesity. Journal of Movement \& Health, 18(1): 1-4. http://dx.doi.org/10.5027/jmh-Vol18-Issue1(2021)art100

Huang, J.F., Wang, X.B. \& Zheng, K.I. (2020). Letter to the Editor: Obesity hypoventilation syndrome and severe COVID-19. Metabolism Clinical and Experimental, 108: 154249. http://doi.org/10.1016/j.metabol.2020.154249

Hudson, G. M. y Sprow, K. (2020) Promoting Physical Activity During the COVID-19 Pandemic: Implications for Obesity and Chronic Disease Management. Journal of Physical Activity and Health, 9: 1-3. https://doi.org/10.1123/jpah.2020-0318

Hull, J.H., Loosemore, M. y Schwellnus, M. (2020). Respiratory health in athletes: facing the COVID-19 challenge. Lancet Respiratory Medicine, 8 (6): 557-228.

https://doi.org/10.1016/S2213-2600(20)30175-2

Revista de Investigación Cuerpo, Cultura y Movimiento

ISSN: 2248-4418 | e-ISSN: 2422-474X | DOI: https://doi.org/10.15332/2422474X

Vol. 11 N. ${ }^{\circ} 1$ | enero-junio de 2021 
Hussain, A., Mahawar, K., Xia, Z., Yang, W. y El-Hasani S. (2020). Obesity and mortality of COVID-19. Meta-analysis. Obesity Research \& Clinical Practice, 14(4):295-300. https://doi.org/10.1016/j.orcp.2020.07.002

Kassir, R. (2019) Risk of COVID- 19 for patients with obesity. Obesity Reviews, 21 (6): e13034. https://doi.org/10.1111/obr.13034

Laddu, D.R., Lavie. C.J., Phillips, S.A y Arena R. (2020). Physical activity for immunity protection: Inoculating populations with healthy living medicine in preparation for the next pandemic. Progress in Cardiovascular Diseases, 64: 102-104 https://doi.org/10.1016/j.pcad.2020.04.006

Maffetone, P.B. y Laursen, P.B. (2020). The perfect storm: Coronavirus (Covid-19) pandemic meets overfat pandemic. Frontiers in Public Health, 8(135), https://doi.org/10.3389/fpubh.2020.00135

Misumi, I., Starmer, J., Uchimura, T., Beck, M. A., Magnuson, T. y Whitmire, J. K. (2019) Obesity expands a distinct population of $\mathrm{T}$ cells in adipose tissue and increases vulnerability to infection. Cell reports, $27(2), 514-524$. https://doi.org/10.1016/j.celrep.2019.03.030

Petrilli, C. M., Jones, S. A., Yang, J., Rajagopalan, H., O'Donnell, L. F., Chernyak, Y., Tobin, K., Cerfolio, R., Francois, F. y Horwitz, L. (2020) Factors associated with hospitalization and critical illness among 4,103 patients with COVID-19 disease in New York City. MedRxiv. https://doi.org/10.1101/2020.04.08.20057794

Ryan, D. H., Ravussin, E. y Heymsfield, S. (2020) COVID 19 and the patient with obesity-the editors speak out. Obesity a research journal, 28 (5): 847. https://doi.org/10.1002/oby.22808

Simonnet, A., Chetboun, M., Poissy, J., Raverdy, V., Noulette, J., Duhamel, A., Labreuche, J., Mathieu, D., Pattou, F. y Jourdain, M., (2020) High prevalence of obesity in severe acute respiratory syndrome coronavirus- 2 (SARS- CoV-2) requiring invasive mechanical ventilation. Obesity a research journal, 28 (7): 1195-1199. https://doi.org/10.1002/oby.23006

Tan, M., He, F.J. y MacGregor, G.A. (2020) Obesity and covid-19: the role of the food industry. British Medical Journal, 369: m2237. https://doi.org/10.1136/bmj.m2237 
Zbinden-Foncea, H., Francaux, M., Deldicque, L. y Hawley, J.A. (2020). Does high cardiorespiratory fitness confer some protection against pro- inflammatory responses after infection by SARS- CoV- 2? Obesity a research, 28 (8): 13781381. https://doi.org/10.1002/oby.22849 